\title{
in Technology
}

\section{Research Paper}

Three Layer Super Learner Ensemble with Hyperparameter Optimization to Improve the Performance of Machine Learning Model

\section{K. T. S. Kasthuriarachchia, ${ }^{\text {,* }}$ and Liyanage S. R ${ }^{\mathrm{b}}$}

a Faculty of Graduate Studies, University of Kelaniya, Dalugama, Kelaniya 11300, Sri

Lanka

b Faculty of Computing and Technology, University of Kelaniya, Dalugama 11300, Sri Lanka

Email correspondence: sanvithat85@gmail.com (K. T. S. Kasthuriarachchi)

Received: 13 March 2021; Revised: 30 March 2021; Accepted: 19 April 2021; Published: 31 May 2021

\begin{abstract}
A combination of different machine learning models to form a super learner can definitely lead to improved predictions in any domain. The super learner ensemble discussed in this study collates several machine learning models and proposes to enhance the performance by considering the final meta- model accuracy and the prediction duration. An algorithm is proposed to rate the machine learning models derived by combining the base classifiers voted with different weights. The proposed algorithm is named as Log Loss Weighted Super Learner Model (LLWSL). Based on the voted weight, the optimal model is selected and the machine learning method derived is identified. The meta- learner of the super learner uses them by tuning their hyperparameters. The execution time and the model accuracies were evaluated using two separate datasets inside LMSSLIITD extracted from the educational industry by executing the LLWSL algorithm. According to the outcome of the evaluation process, it has been noticed that there exists a significant improvement in the proposed algorithm LLWSL for use in machine learning tasks for the achievement of better performances.
\end{abstract}

Keywords: Classifier, ensemble, feature selection, hyperparameter, optimization, random search, super learning 


\section{Introduction}

Rating the scores and early prediction of domain specific data has become very important in many research contexts. Most prediction tasks can be conducted by utilizing different machine learning algorithms. Prediction algorithms can be categorized based on their predictive tasks. Decision tree classifier, Random Forest classifier, Naïve Bayes analyzer, Artificial Neural Network, Linear Regression, Logistic Regression, Support Vector Classifier and K-Nearest Neighbor classifier are several such machine learning mechanisms designated to perform classification, clustering, association rule mining etc. A substantial number of research studies have been carried out in various subject domains with these machine learning algorithms to provide predictive and analytical decisions [23].

The choice of the algorithm, most appropriate for a given dataset, is not a trivial task since that decision influences the overall accuracy of the predicted model [21]. Generally, researchers would compare the performance of selected algorithms on a test data set and select the algorithm that statistically outperforms the other algorithms in a significant manner $[27,32]$. However, there is the uncertainty whether the selected algorithm will be the best for all possible real-world datasets. As stated in the "No free lunch theorem" the computational cost of finding a solution, averaged over all problems in the class, is the same for any solution method. Recently, methods such as boosting and bagging have outperformed a single best classifier when predicting on real world datasets [38]. Therefore, when none of the algorithms significantly outperforms other methods it is pragmatic to select a few algorithms and to determine the best during runtime [12].

Ensemble methods are more apt for the use in similar situations where the researcher is able to develop various models using different machine learning algorithms to a selected data set and combine into a single classifier. Many prediction algorithms can be collated on a single model to construct an ensemble. This ensemble is constructed to reduce 
bias and variance [11]. The super learner is an ensemble machine learning method which could combine several machine learning models and configure to produce a single predictive model for efficient and robust predictions. Data analyzer cannot strictly prioritize a specific machine learning technique, rather they can select several techniques.

This study focuses on implementing a priori-specified hyper parameterized ensembling machine learning approach which combines several machine learning algorithms into a single algorithm and returns a prediction method with the best cross validated Log Loss Error (CLLE). The individual modeling techniques of K-Nearest Neighbor (KNN), Random Forest (RF), Naïve Bayes (NB), Support Vector Machine (SVM), Decision Tree (DT) and Logistic Regression (LR) approach-based sensitivity estimation were used. Hyperparameters refer to parameters whose values are set by the user before training the algorithm. They affect the entire ensemble's performance structure and the complexity [43]. Super learner algorithm occupies a set of candidates learning algorithms and apply them to a data set to choose the optimal learner or learner combination so that it will perform better than other learners [21].

This paper is motivated to discuss the proposed super hyperparameterized ensembling machine learner in three aspects. First, the implementation of the new learner with the weights, then the evaluation procedure of the learner on predicting tasks using different datasets from a variety of educational disciplines and finally, focus on using this novel super learner in future applications by optimizing the parameters. The paper is organized as follows. Section 2 presents the existing problems of super learner ensemble and the approaches followed by previous studies to overcome it by using hyperparameter optimization. Section 3 presents the methodology followed to implement the super learner ensemble with random hyperparameter optimizer. Finally, section 4 presents a comprehensive discussion about 
the experiments with results and validation of the ensemble against deep learning Convolutional Neural Network.

\section{Background}

The ensembles are of three types. Bagging, Boosting, Blending/ Stacking. Bagging and boosting are two of the common ensemble techniques used in machine learning. Bagging is the method for generating multiple versions of predictors and form an aggregated predictor by voting each version and getting the average of them [7]. Bagging meta-estimator and the random forest are the algorithms followed bagging approach. Boosting works in a similar way to bagging by combining several poor performing base learners in an adaptive way. Experimental work showed that bagging is effective for data sets with noisy values [39]. In boosting, the learning algorithms are given different distribution or weighting according to the errors of the base learners [8, 31]. AdaBoost, Gradient Boosting (GBM), eXtream Gradient Boosting (XGBM), Light GBM, and CatBoost are considered as Boosting techniques. The third approach is blending/stacking which takes the output of selected base learners on the training data and applies another learning algorithm on them to predict the response values [22]. Stacking uses a first- level ensemble of classifiers to form the second- level meta -input [45]. Research experiments have shown that the stacking has better robustness properties [10]. Super learner is an application of stacked generalization approach.

The main research task incorporated into this article is the implementation of a super learner ensemble with high accuracy and low computational time. Super learning concept could be easily adopted to any domain where an analytical model needed to be generated. Accordingly, the super learner ensemble is implemented by dividing the tasks into two steps. First, the base learner selection through weighted prediction scoring. Second, optimize the hyperparameters used by the super learner ensemble to obtain the best prediction accuracy in minimal duration. This section discusses the 
background details, characteristics, current research contribution, drawbacks and advantages of each important area of study.

\section{Super Learning}

It is in fact impractical to identify a priori the machine learning algorithms use in the regression of classification problem in decision making. This leads the data analyst to use many different machine learning algorithms to develop different models and evaluate the performance of them using resampling by cross validation. Once the evaluation is performed many times in different configurations, the best out of the all models is selected to predict the target attribute and make decisions. A single machine learning algorithm may be unable to capture the complete underline structure of the data to derive optimal predictions. This is where the integration of multiple models were gathered into a single meta - model [40]. The main intuition behind the concept of Super learning is to address the point which "Why all the prediction models are not considered and select the best model out of all for the machine learning problem". It is a fact that the super learner ensemble approach encourages to collate different machine learning models (base learner models) and construct a single predictive model (meta- model) with high predictive performance. Super learner concept was first introduced by a set of researchers in a biological study. This is an application of stacked generalization to $\mathrm{k}$ - fold cross validation since all the analysis models use the same k-fold splits of the data and a meta- model fits into the out-of-fold predictions of each of the models. The traditional machine learning approaches build a single hypothesis based on the training data but, the ensemble approach attempts to develop a set of hypotheses and combine them to form a new hypothesis [36]. Different studies were carried out based on the super learning concept. Once multiple prediction models are combined, more information could be captured in the fundamental structure of the data [8]. A researcher highlighted the importance of recognizing the uncertainty when selecting models, and the prospective role of 
assembling can play in combining several models to create one that outperforms single models [41]. In a study about improving accuracy and reducing variance of behavior classification in accelerometer done by researchers has shown that super learning can be easily adapted to any type of industry to achieve better accuracy in the predicted model [21]. Also they emphasized the importance of the human intervention and the computation time required to implement a super learner for the machine learning tasks. The ensemble learning performs better than the individual base learners [36]. A researcher has pointed out that the high computational time and the memory requirement for the smooth execution of super learner approach is significant and thereby, super learner can be potentially flawed too [35].

\section{Base Classifiers}

The proposed super learner ensemble has base classifiers and a metaclassifier. (1) The base classifier/ learner fits the dataset using different machine learning algorithms. KNN, RF, NB, SVM, ANN and DT were used as base learners in this study. (2) The meta- learner is fitted on the predictions of the base learners. LR algorithm is used as the metalearner in forming the super learner. The following section provides a brief description of the base learners and the meta-learner incorporated to this study. KNN is the most simple and straight forward data mining algorithms [34]. $\mathrm{K}$ is the number of nearest neighbors that are used to make the prediction and it calculates the distance between data points using the Euclidean distance measurement [9]. RF gives more precise predictions even for a large sample size. It captures the discrepancy of several input variables at the same time and allows high number of observations to participate in the prediction [30]. NB simplifies the calculation of probabilities by assuming that the probability of each attribute of a given class value is independent of all other attributes [2]. SVM used for both classification and regression tasks. The SVM algorithm plots the data items as a point in ndimensional space regarding the number of features in the dataset. 
Then each feature is represented in a particular coordinate in $\mathrm{x}$ and $\mathrm{y}$ axis. Then, the classification is performed by finding the hyper-plane by differentiating the classes successfully [15]. DT algorithm uses to select attributes of the data set based on the information gain measurement which is known as entropy of the attribute. The attributes which have the high information gain value and high gain ratio value will be selected for splitting the attributes [18]. ANN is a machine learning algorithm which is modeled for data handling and especially useful for distinguishing the key relationships among an arrangement of factors or patterns in the data [1].

\section{Meta Classifier}

\section{Logistic Regression}

Logistic regression is used to describe data and the relationship between one dependent binary variable and one or more nominal, ordinal, interval or ratio level independent variables. The classified observations and a low percentage of misclassified observations [32]. This model is frequently used in situations where more predictor variables are considered in the analysis [37].

\section{Hyperparameter Optimization}

The parameters which define the model architecture are known as hyperparameters. The process of identifying the ideal model architecture is done in order to change these parameter values and measures the performance attributes of the predicted model is known as hyperparameter tuning or hyperparameter optimization. This is achieved in three different methods, namely; (1) Grid search (2) Random search and (3) Bayesian optimization. Grid search is the most basic method. The prediction model will be created for each possible combination of all the hyperparameter value and will evaluate each model and select the architecture which produces the best result. Random search finds better models by effectively searching a larger, less promising configuration space than grid search method [14]. The next method, bayesian optimization is also called the surrogate method 
which keeps track of past evaluation results which are used to form a probabilistic model, maps the hyperparameters to a probability of a score on the objective function that it uses. It could find a better set of hyperparameters in less time because they study about the best set of hyperparameters to evaluate, based on past trials.

Researchers have focused on this hyperparameter optimization in different forms. A researcher has done a study to find a method to accelerate the search process by transferring information from previous trials to other datasets [4]. The key challenge they faced was the accuracy measurement. It was a relatively difficult task to maintain the accuracy of the model while maintaining the speed of the analysis through hyperparameter tuning [46]. Another team of researchers introduced a systematic framework to build ensembles with optimal weights for regression problems [33]. They were able to find the optimized ensemble weights that minimize both bias and variance of the predictions while tuning the hyperparameters of the base learners. A study about the use of baysian optimization to hyperparameter tuning in ensemble learning has been mentioned in a study and they could build an optimized strategy to exploit trained models and improved ensembles to use as a classifier at the lower cost of regular hyperparameter optimization [17]. It could be observed that the existing ensemble techniques consider the base model construction and the weighted averaging to be independent steps. However, the researchers concluded that combining these two components will lead to a low performing ensemble learner. A study has proposed a weighted ensemble approach by assigning estimated weights in order to obtain more accurate ensemble result than the classical ensemble [33]. Another researcher has introduced a probabilistic ensemble weighting approach used on cross-validation for hyperparameter optimization [22].

There have not been studies where the base learner had been modeled with hyperparameter optimization and the model weight assignment 
for the implementation of an optimal ensemble learner in achieving the optimal accuracy of the prediction.

\section{Materials and Methods}

The implementation of the novel super learner is carried out in two phases named; (1) Log loss weighted prediction and (2) Hyperparameter optimization. The entire structure of the proposed super learner named; Log Loss Weighted Super Learner Model (LLWSL). The LLWSL is represented using a pseudocode as shown in below Figure 1.

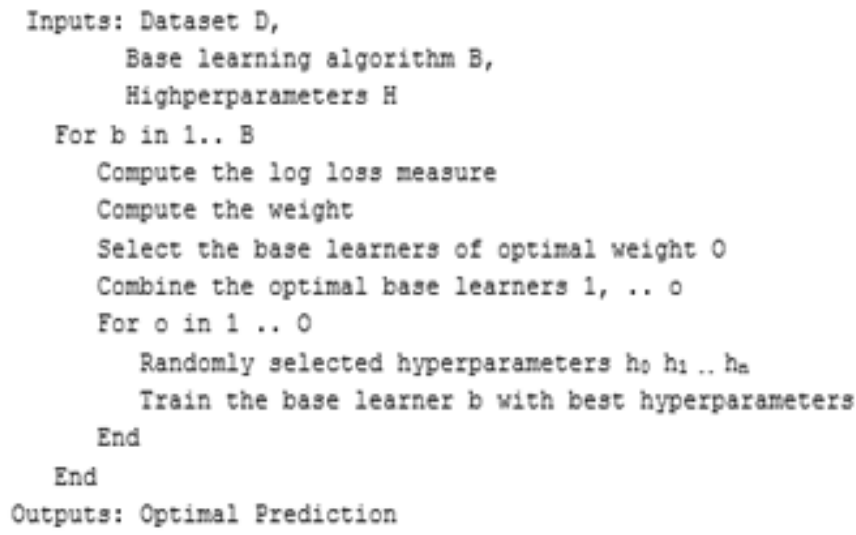

Figure 1. Pseudocode of Log Loss Weighted Super Learner Model (LLWSL)

The proposed super learner approach consists of base classification with KNN, RF, NB, SVM, ANN and DT algorithms and the metaclassifier used by the super learner model is derived using logistic regression technique. The reason for the selection of different algorithms is that they are following significantly different approaches for the model generation and focus on the data in different aspects to make a significant contribution to ensemble implementation. The base learners derive the prediction models on the provided dataset. The initial set of hyperparameters were identified and they have been given values priori. The log losses of each model were recorded and random weight values varying between ' 0 ' and ' 1 ' are assigned to them while the base learner prediction models are developed. In a study on ensemble implementation using log loss function and it was derived 
the predicted model with some considerable level of performance though the hyperparameter tuning has not been done [3]. After a comparison of the weights of optimal base learners were selected. Those base learners were extracted and fed into random search hyperparameter tuning approach. The implementation of the super learner ensemble model is illustrated in Figure 2.

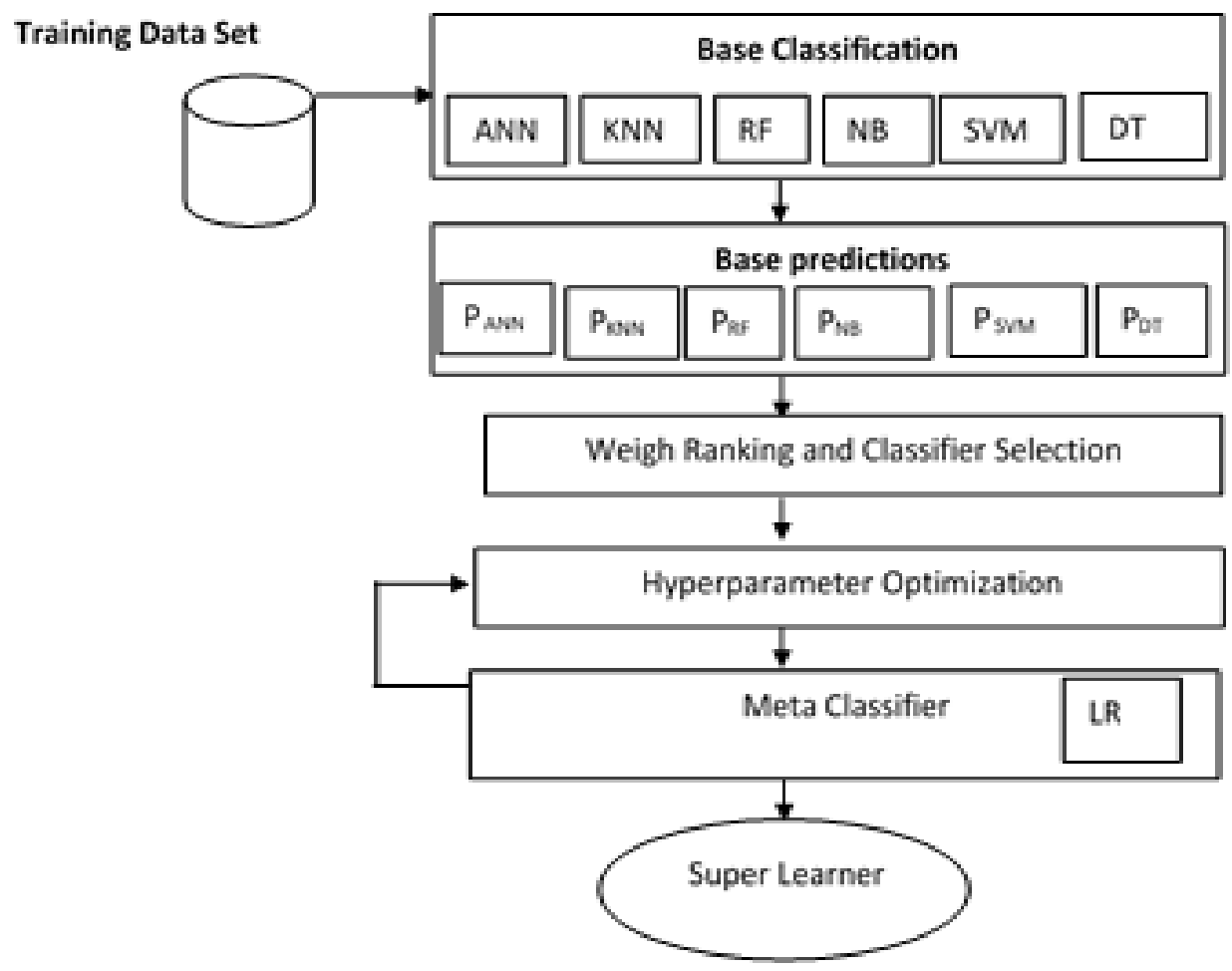

Figure 2. The architecture of Optimal Super Learner Ensemble.

\section{Log Loss Weighted Prediction Model}

In order to measure the error, one scores the models by assigning weights to the base classifiers, it's the likelihood function known as the log loss. Log loss is based on probabilities. Instead of maximizing accuracy of the model, the error will be minimized by this technique. The lower the log loss, higher is the model accuracy. Therefore, the logloss is selected as the benchmark for comparing multiple prediction models. 
The proposed optimization model uses to derive the best combination of base learners by measuring weighs through their log loss values and rate the models with weights. Then one aggregates the models of nonzero weights together to form the input to the meta- learner, which functions based on logistic regression. The log loss function of a machine learning model could be given as follows;

$$
\text { LogLoss }=-1 / \mathrm{n}>\left[y_{i} \cdot \log _{\theta}\left(y_{i}\right)+\left(1-y_{i}\right) \cdot \log _{\theta}\left(1-y_{i}\right)\right] \quad \text { Equation } 1
$$

Where, $\mathrm{n}$ is the number of instances in the dataset, $\mathrm{y}$ is the dependent variable in the dataset which will be either 0 or $1, y_{i}$ is the model probability of assigning label $j$ to instance $i$. Based on the weights of each model, a random weight number is assigned to every model. Then the best classifiers are selected to hyperparameter tuning.

\section{Hyperparameter Optimization in Super learner Ensemble}

The hyperparameters were identified by performing the random hyperparameter search approach. There was a study on finding the best hyperparameter tuning method for ensemble learning by the random search approach was selected as the best [14]. The Hyperparameters that could be used to optimize are already given their initial values. Once the optimal models are selected, in the first stage, they have been fed to the meta-learner which is developed by a logistic regression to construct the super learner. During this, the random search hyperparameter optimization was performed by setting the log loss as the scoring method. The model accuracy and the speed of the super learner ensemble execution was recorded. The hyperparameters which enable that performance of the super learner were also recorded.

\section{Data Sets}

The evaluation of proposed approach has been done using a dataset taken from the education domain named LMSSLIITD. The LMSSLIITD data set mainly has two categories of data. (1) Class room teaching data, and (2) LMS usage data. Classroom data was gathered by 
distributing a structured questionnaire among the university students who were enrolled in an Information Technology degree program. The second, LMS data set was collected by accessing the MOODLE data of a course module offered for thirteen weeks of an Information Technology degree program in a Sri Lankan university. There are 170 instances and 20 variables in the classroom teaching data set and 799 instances with 11 attributes in the LMS data set. Table 1 illustrates the data gathered from the Learning Management System.

Table 1. Description of the LMS data in the LMSSLIITD data set.

\begin{tabular}{|c|c|c|}
\hline Variable & Description & Value \\
\hline Student_id (w1) & Id number of the student & \\
\hline Gender (w2) & Gender of the student & male -1 , female 2 \\
\hline $\begin{array}{l}\text { Total_cliks_per_day } \\
\text { (w3) }\end{array}$ & $\begin{array}{l}\text { Total number of times a student hits on the } \\
\text { material in a day }\end{array}$ & \\
\hline $\begin{array}{l}\text { Highest_education } \\
\text { (w4) }\end{array}$ & The level of education of the student & $\begin{array}{l}\mathrm{A} / \mathrm{L}-1, \text { Associated } \\
\text { diploma }-2, \\
\text { diploma }-3\end{array}$ \\
\hline Age_range (w5) & Age range of the student & $\begin{array}{l}(18-25)-1,(25- \\
30)-2\end{array}$ \\
\hline Repeat_module (w6) & $\begin{array}{l}\text { Number of times the studemt has } \\
\text { attempted the modle }\end{array}$ & \\
\hline Studied_credits (w7) & Total credits obtained by the student & \\
\hline Disabilities (w8) & Whether student has a disability or not & No -0, Yes -1 \\
\hline Date_submitted (w9) & $\begin{array}{l}\text { The date of assignment submission from } \\
\text { the day the assignment is given to attempt. }\end{array}$ & \\
\hline Score (w10) & Marks scored for the assignment & range $0-100$ \\
\hline Final_result (w11) & Final result of the module & Fail - 0, Pass - 1 \\
\hline
\end{tabular}


Table 2. Description of the Classroom data in the LMSSLIITD data set.

\begin{tabular}{|c|c|c|}
\hline Variable & Description & Value \\
\hline Age (w1) & Age of the student & \\
\hline Gender (w2) & Gender of the student & (male -1 , female 2$)$ \\
\hline Location (w3) & $\begin{array}{l}\text { The place where the student stay during } \\
\text { the study period }\end{array}$ & $\begin{array}{l}\text { Home }-1 \text {, House of a relation - } \\
2 \text {, boarding house }-3 \text {, } \\
\text { university hostel }-4\end{array}$ \\
\hline $\begin{array}{l}\text { Reason selection } \\
\text { (w4) }\end{array}$ & The reason for selecting degree course & \\
\hline AL field (w5) & $\begin{array}{l}\text { The field of study in university entrance } \\
\text { AL exam }\end{array}$ & $\begin{array}{l}\text { Biology }-1 \text {, Maths }-2 \text {, } \\
\text { Commerce }-3 \text {, Arts }-4 \text {, } \\
\text { Technology }-5\end{array}$ \\
\hline $\begin{array}{l}\text { AL English Grade } \\
\text { (w6) }\end{array}$ & $\begin{array}{l}\text { The grade obtained for English paper in } \\
\text { AL }\end{array}$ & A-1, B- 2, C-3, S-4, F-5 \\
\hline $\begin{array}{l}\text { OL English Grade } \\
\text { (w7) }\end{array}$ & $\begin{array}{l}\text { The grade obtained for English paper in } \\
\text { OL }\end{array}$ & A-1, B-2, C-3, S- 4, W- 5 \\
\hline $\begin{array}{l}\text { Extra Activities } \\
\text { (w8) }\end{array}$ & $\begin{array}{l}\text { Whether the student has done extra- } \\
\text { curricular activities done during the study } \\
\text { period }\end{array}$ & No -0, Yes -1 \\
\hline $\begin{array}{l}\text { Society } \\
\text { membership (w9) }\end{array}$ & $\begin{array}{l}\text { Whether the student has hold any } \\
\text { memberships in university societies }\end{array}$ & No -0 , Yes -1 \\
\hline Social Net (w10) & Whether the student used social networks & No -0 , Yes - 1 \\
\hline Politics (w11) & The involvement to political activities & No -0 , Yes - 1 \\
\hline Attendance (w12) & Rate the attendance to lectures & $\begin{array}{l}\text { Below } 50 \%-1,51-74 \%-2,75- \\
94 \%-3 \text {, above } 95-100 \%-4\end{array}$ \\
\hline Hours (w13) & $\begin{array}{l}\text { Number of hours spend for studies per } \\
\text { day }\end{array}$ & $\begin{array}{l}\text { Below } 1-1,(1-3)-2,(3-6)- \\
3,(6-10)-4 \text {, more than } 10-5 \text {, } \\
\text { never - } 6\end{array}$ \\
\hline $\begin{array}{l}\text { Scholarships } \\
\text { (w14) }\end{array}$ & Whether the student won scholarships & No -0 , Yes -1 \\
\hline $\begin{array}{l}\text { Perception } \\
\text { teaching (w15) }\end{array}$ & $\begin{array}{l}\text { The perception of student on teaching in } \\
\text { the university }\end{array}$ & $\begin{array}{l}\text { Highly satisfy }-1 \text {, Satisfy }-2 \text {, } \\
\text { Moderate }-3 \text {, Dissatisfy }-4 \text {, } \\
\text { Highly dissatisfy - } 5\end{array}$ \\
\hline $\begin{array}{l}\text { Avg family } \\
\text { income(w16) }\end{array}$ & The average family income & $\begin{array}{l}\text { Below } 25000-1,(25000- \\
30000)-2,(30000-50000)-3, \\
(50000-80000)-4,(80000- \\
100000)-5,(100000-200000)- \\
6, \text { above } 200000-7\end{array}$ \\
\hline $\begin{array}{l}\text { Batch } \\
\operatorname{missed}(\text { w17) }\end{array}$ & $\begin{array}{l}\text { Whether the student has postponed the } \\
\text { studies }\end{array}$ & No -0, Yes -1 \\
\hline $\begin{array}{l}\text { Repeat } \\
\text { modules(w18) }\end{array}$ & $\begin{array}{l}\text { Whether the student has any repeat } \\
\text { modules }\end{array}$ & No -0, Yes -1 \\
\hline Internship(w19) & $\begin{array}{l}\text { Whether the student followed any } \\
\text { industry internship }\end{array}$ & No -0 , Yes -1 \\
\hline CGPA(w20) & Final grade of the student & $(3-4)-1,(2-3)-2$, below $2-3$ \\
\hline
\end{tabular}




\section{Data Preparation and Feature Extraction}

Knowledge is extracted from data by following a series of steps. This is known as Knowledge Discovery in Databases (KDD). Figure 3 illustrates the steps of KDD process.

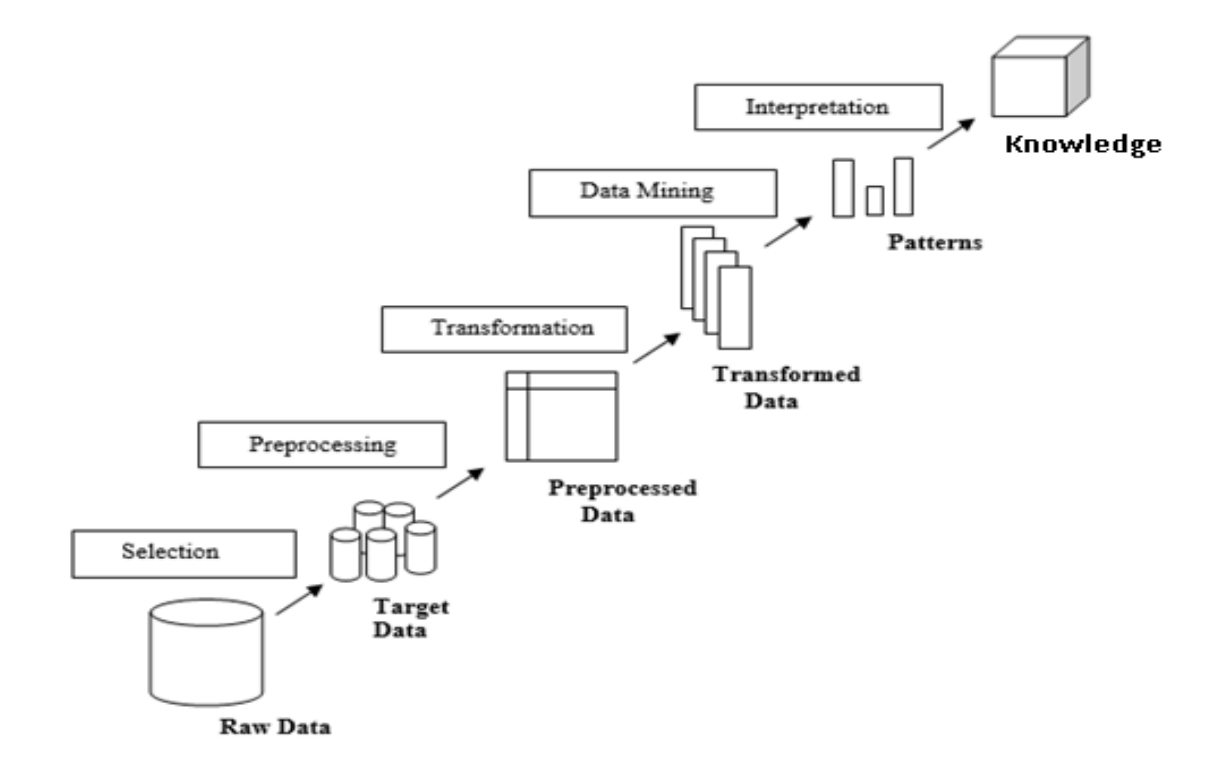

Figure 3. Steps of KDD process.

The preparation of data is an important step before starting the actual data analysis. When the data is collected from questionnaires or surveys, they should be recorded electronically and should be transformed into a suitable format for the analysis. The data is preprocessed. The feature selection step was carried out to both data sets in LMSSLIITD [42]. The sensitive variables were selected from both datasets using the boruta package in Python and the variable importance is graphed as illustrated in Figure 4 and Figure 5 [19]. According to the feature selection high important variables of both datasets are extracted. 


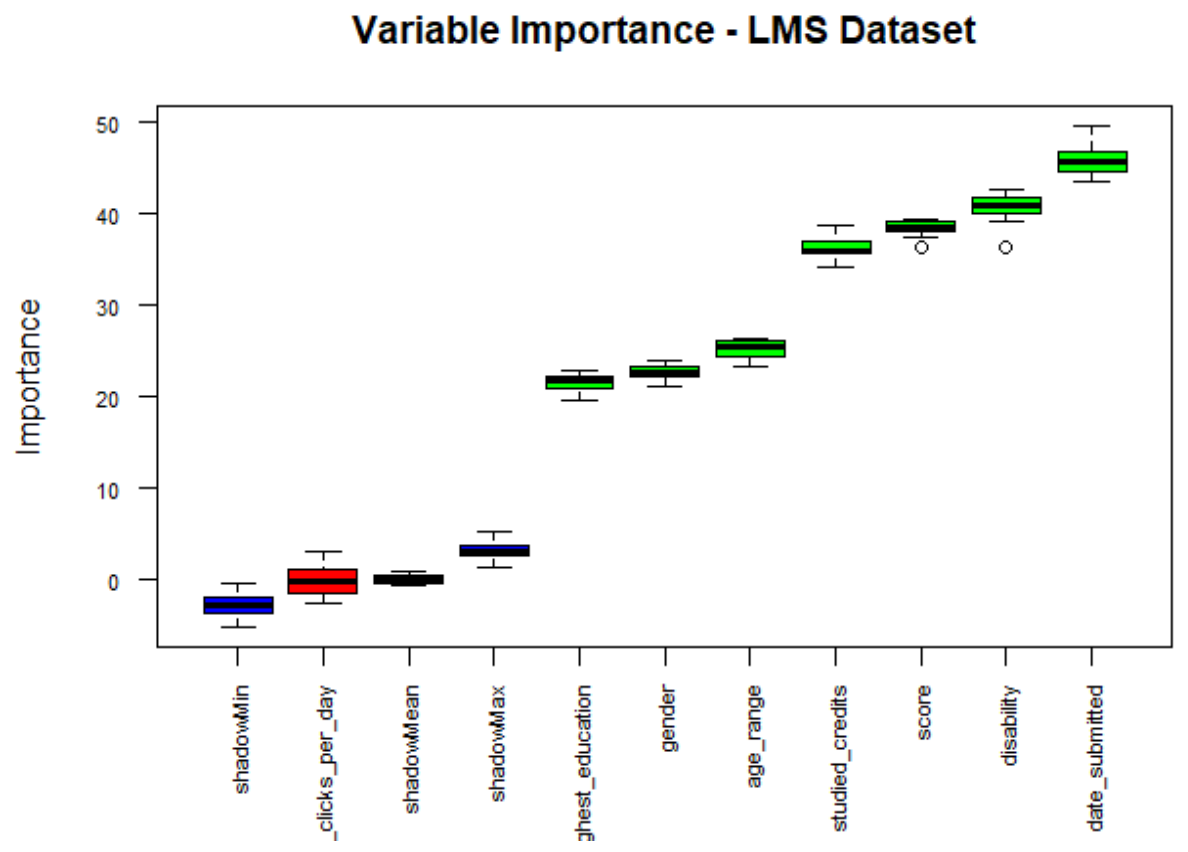

Figure 4. The LMS Dataset Features Selected by Boruta.

Variable Importance - Class room Dataset

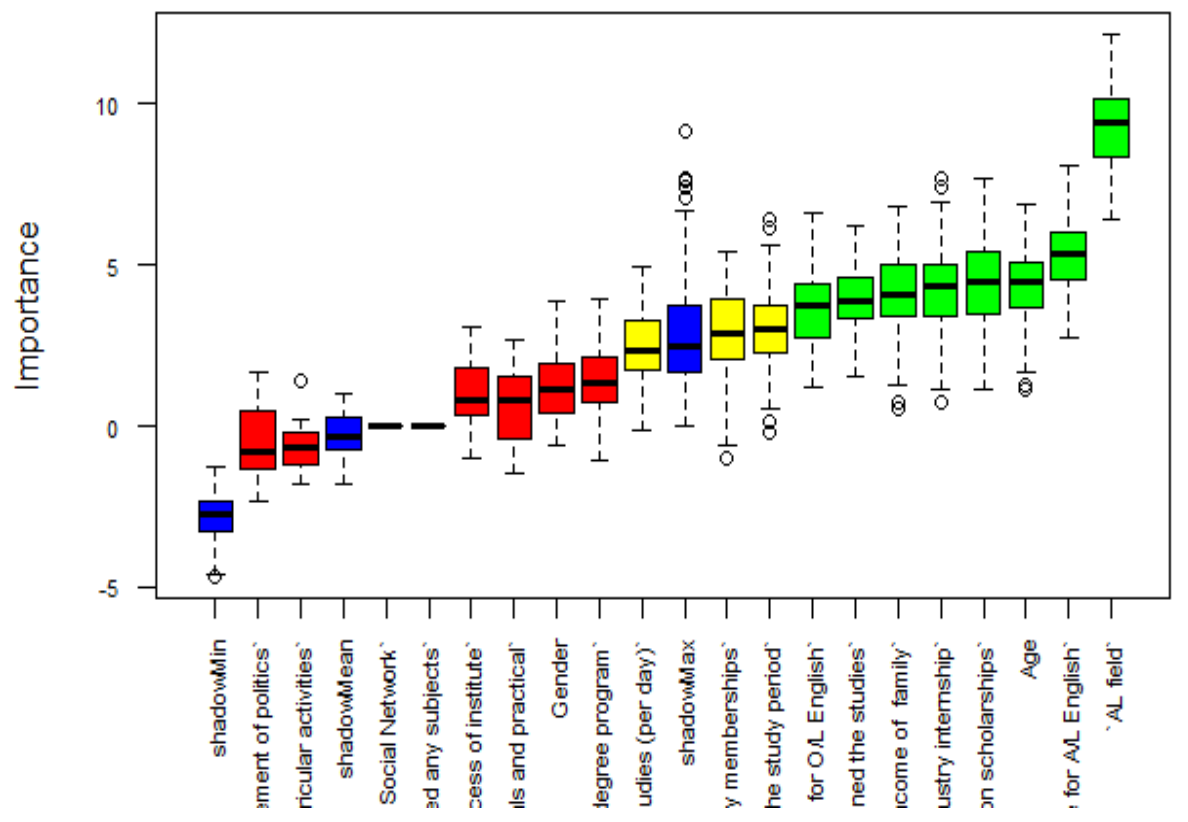

Figure 5. The Classroom Dataset Features Selected by Boruta. 


\section{Performance Evaluation of LLWSL algorithm}

The proposed novel algorithm is applied to the datasets mentioned in above and the accuracy rate derived for each is recorded. The derived results are compared against the accuracies of the datasets given before apply the proposed algorithm. The time taken for both situations are also recorded to see the complexity of the proposed algorithm.

\section{Model Evaluation}

The results of the experiment is statistically validated using a hypothesis test and evaluated the model performance to prove the suitability of the proposed approach for the data mining tasks.

\section{Results and Discussion}

In the designing of the super learner ensemble six machine learning algorithms were taken for the base classifier designing. In order to optimize the super learner, the proposed LLWSL algorithm was used. Priori, the super learner ensemble was constructed by combining the base learners. Their performances were recorded as given by Table 3 .

Table 3. Performance of the Base Learners and the Super Learner Ensemble

\begin{tabular}{|l|c|c|c|c|c|c|}
\hline \multirow{2}{*}{$\begin{array}{l}\text { Machine Learning } \\
\text { Algorithm }\end{array}$} & \multicolumn{3}{|c|}{ LMS Data } & \multicolumn{3}{c|}{ CLASS ROOM DATA } \\
\cline { 2 - 7 } & $\begin{array}{c}\text { Prediction } \\
\text { Accuracy } \\
(\%)\end{array}$ & $\begin{array}{c}\text { Accuracy } \\
\text { Variation } \\
(+/-)\end{array}$ & $\begin{array}{c}\text { Log } \\
\text { Loss }\end{array}$ & $\begin{array}{c}\text { Prediction } \\
\text { Accuracy } \\
(\%)\end{array}$ & $\begin{array}{c}\text { Accuracy } \\
\text { Variation } \\
(+/-)\end{array}$ & $\begin{array}{c}\text { Log } \\
\text { Loss }\end{array}$ \\
\hline Decision Tree & 78 & 17 & 0.021 & 65 & 10 & 15.350 \\
\hline Random Forest & 81 & 16 & 0.027 & 70 & 13 & 0.4252 \\
\hline Naïve Bayes & 82 & 10 & 0.901 & 62 & 16 & 0.7669 \\
\hline K- Nearest Neighbor & 75 & 14 & 0.863 & 60 & 12 & 15.350 \\
\hline $\begin{array}{l}\text { Support Vector } \\
\text { Machine }\end{array}$ & 79 & 9 & 0.467 & 68 & 13 & 0.6356 \\
\hline $\begin{array}{l}\text { Artificial Neural } \\
\text { Network }\end{array}$ & 81 & 0 & 0.500 & 63 & 16 & 0.6368 \\
\hline $\begin{array}{l}\text { Super Learner } \\
\text { Ensemble }\end{array}$ & 80 & 15 & 0.021 & 65 & 10 & 0.9147 \\
\hline
\end{tabular}


The 10 fold cross validation was used as the validation method. The entire process was repeated 5 times. The number of iterations for random search was selected as 10 . The Randomized SearchCV package from Scikit-learn library was used to perform the hyperparameter tuning task [26]. The Sequential Least Square Programming Algorithm (SLSQP) from Phython SciPy optimization library was used to solve the optimization problem [16]. After applying the LLWSL for the optimization, the prediction accuracies and the log loss values of the super learner ensemble were recorded and illustrated in Table 4.

Table 4. Comparison of the Super Learner performance before and after LLWSL

\section{Before LLWSL}

Data Set Prediction Accuracy Construction Prediction Accuracy Variation Time (ms) Accuracy
(+) -)
80

65

65

15

10 $(\%)$

85

17.66

12.87

AFTER LLWSL

Accuracy Construction Variation Time $(\mathrm{ms})$

Class room

Data

(10)

According to the figures provided in Table 4, the prediction accuracy of super learner ensemble, after performing LLWSL has shown a significant improvement in terms of the accuracy level for both datasets. The execution times of both cases show that the super learner has a significant improvement from $17.66 \mathrm{~ms}$ to $14.18 \mathrm{~ms}$ in LMS dataset and from 12.87 to 4.56 in classroom dataset. The LLWSL algorithm has been applied to increase the performance of the entire ensemble by optimizing the parameters of the selected classifiers too. Once the ensemble was created, the tuned hyperparameters in both executions have been listed in the Table 5 . 
Table 5. Optimized Hyperparameters of each Base Learner in the Super Learner.

\begin{tabular}{|c|c|c|c|c|}
\hline \multirow{2}{*}{$\begin{array}{l}\text { Machine Learning } \\
\text { Model }\end{array}$} & \multicolumn{2}{|l|}{ LMS DATASET } & \multicolumn{2}{|c|}{ CLASS ROOM DATASET } \\
\hline & Hyperparameter & Value & Hyperparameter & Value \\
\hline Decision Tree & max_depth & 2 & max_depth & 10 \\
\hline \multirow[t]{4}{*}{ Random Forest } & n_estimators & 8 & n_estimators & 5 \\
\hline & min_samples_split & 9 & min_samples_split & 10 \\
\hline & max_features & 7 & max_features & 7 \\
\hline & max_depth & None & max_depth & 10 \\
\hline K- Nearest Neighbor & n_neighbors & 50 & n_neighbors & 50 \\
\hline \multirow{3}{*}{$\begin{array}{c}\text { Support Vector } \\
\text { Machine }\end{array}$} & svc_kernel & $\mathrm{rbf}$ & svc_kernel & $\mathrm{rbf}$ \\
\hline & & & & \\
\hline & svc_class_weight & None & svc_class_weight & None \\
\hline $\begin{array}{c}\text { Artificial Neural } \\
\text { Network }\end{array}$ & Hidden_layer_sizes & 50 & Hidden_layer_sizes & 50 \\
\hline $\begin{array}{l}\text { Super Learner } \\
\text { Ensemble }\end{array}$ & Classifier_C & 20 & Classifier_C & 10 \\
\hline
\end{tabular}

\section{Model Evaluation}

The Receiver Operator Characteristic curves (ROC) are generated to illustrate the diagnostic ability of the Super Learners before and after applying LLSWL algorithm. The ROC curve shows the trade-off between sensitivity and specificity [6]. One of the most common approaches to summarize the performance of classifiers is the calculation of area under the ROC curve (AUC). The ROC curves were generated in the study to illustrate the performance of the super learner models.

According to the Figure 6, the left ROC illustrates the AUC value of the test before applying LLWSL and right side ROC illustrates the AUC after applying LLWSL. After LLWSL, the AUC is, 0.92 and it is greater than the previous LLWSL value which is 0.67 . After enhancing the super learner, the model derived a better AUC value which is closer to 1. 
As figure 6 illustrates, the left ROC was derived before applying LLWSL and right side ROC was derived after applying the LLWSL. In this situation also, the result was better than the previous test and closer to 1 . The AUC values of both figures prove that the super leaner model has been enhanced with better performance once the proposed LLWSL algorithm is applied.
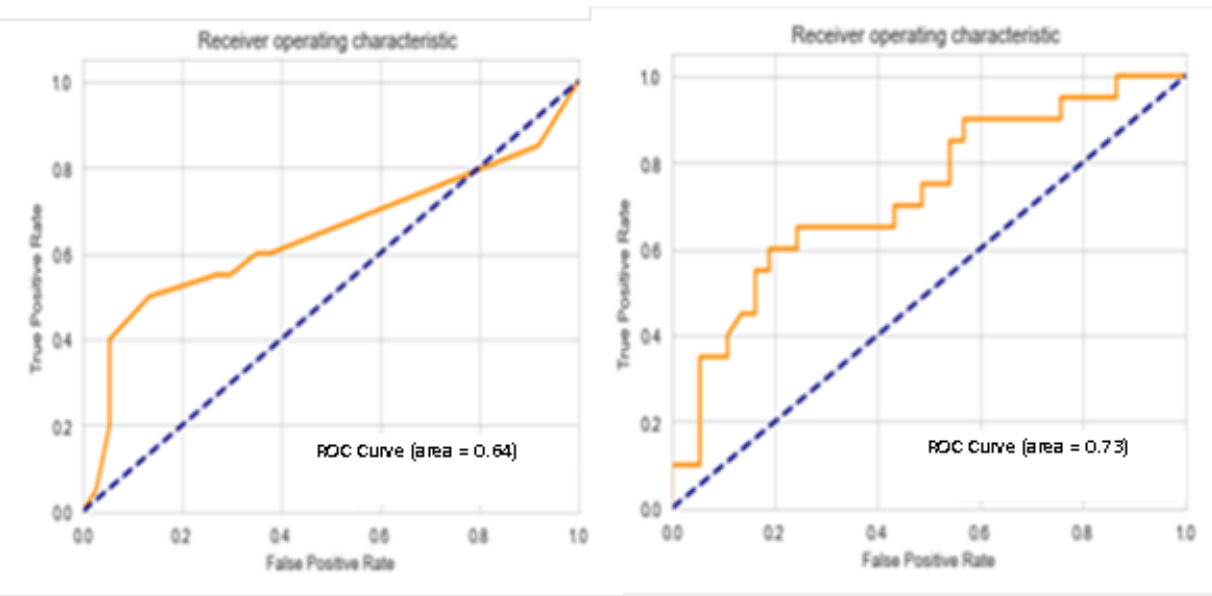

Figure 6. ROC Curve Area of the Super Learner before and after LLWSL derived by Classroom Dataset.

Once the machine learning tasks and the super learner ensemble are created, the derived models have to be validated and evaluate the performances. The measurement of the performance of machine learning models in statistics is different from normal data model validation [20]. The most common statistical hypothesis test method used to test the difference of results between two machine learning algorithms over different data sets is parametric statistical test called, a paired t-test. This evaluates whether the average difference of the algorithm performance over the data sets is significantly different from [41]. Therefore, considering the situation before applying the LLWSL algorithm and after applying LLWSL algorithm, each of the classifier was tested against the super learner using a paired student's t-test combined via random subsamples of the training dataset.

Accordingly, first, the super learner ensemble and random forest classifier were considered for both before applying LLWSL, and after 
applying LLWSL. The test commenced once the null and alternative hypotheses were made.

The paired student's t- test is assumed that the data used to perform the test should be sampled independently from the two populations being compared [29]. However, since the data in the training and testing sets are overlapped in different iterations, the independence assumption is violated. As a solution, researchers of a study have suggested a novel variance estimation to compute the P-value and embedded it to paired student's t-test [49].

Before LLWSL on LMS dataset, the null hypothesis $\left(\mathrm{H}_{0}\right)$ assumed that both models perform the same and alternative $\left(\mathrm{H}_{1}\right)$ assumed that the models perform differently.

$\mathrm{H}_{0}$ : There is no difference between the performance of the super learner ensemble and the Random Forest classifiers before applying LLWSL.

$\mathrm{H}_{1}$ : There is a difference between the performance of the super learner ensemble and the Random Forest classifiers before applying LLWSL.

Once the paired student's t- test is performed, it has been observed that the p-value was about 0.458 , which exceeded the standard significant level of $0.05(0.458>0.05)$. This implies that the null hypothesis cannot be rejected, and it has been statistically convincing evidence that random forest and super learner ensemble perform almost a similar prediction and no difference between them before applying LLSWL on LMS data was reported. Similar steps were followed after LLWSL as well. The null and alternative hypotheses are:

$\mathrm{H}_{0}$ : There is no difference between the performance of the super learner ensemble and the Random Forest classifiers after applying LLWSL.

$\mathrm{H}_{1}$ : There is a difference between the performance of the super learner ensemble and the Random Forest classifiers after applying LLWSL.

After the statistical test, the p-value was about 0.0486 , which is below the significant level of 0.05 . This implies that the null hypothesis is rejected, and it has been statistically proven that the random forest and super learner ensemble performed differently after applying LLWSL on 
LMS data. This is a positive finding on the performance of the super learner ensemble. Once the LLWSL algorithm is applied and the super learner is tuned to perform better, it could execute the machine learning task and could show a significantly different result than the result if only the Random Forest algorithm had been used. Further, this statistical test implies that rather than selecting only the Random Forest algorithm for the prediction task, the super learner ensemble could be used to achieve better performance. This procedure has been iteratively performed for all six algorithm pairs of both datasets. The obtained pvalues were recorded and illustrated in Table 6.

Table 6. P- Values obtained after Paired student's T-Test.

\begin{tabular}{lcccc}
\hline & \multicolumn{3}{c}{ P - Value for Comparison } \\
\cline { 2 - 5 } Machine Learning Algorithm Pair & \multicolumn{2}{c}{ Before LLWSL } & \multicolumn{2}{c}{ After LLWSL } \\
\cline { 2 - 5 } & $\begin{array}{l}\text { LMS } \\
\text { Dataset }\end{array}$ & $\begin{array}{l}\text { Classroom } \\
\text { Dataset }\end{array}$ & $\begin{array}{l}\text { LMS } \\
\text { Dataset }\end{array}$ & $\begin{array}{l}\text { Classroom } \\
\text { Dataset }\end{array}$ \\
\hline $\begin{array}{l}\text { Super Learner ensemble and Random } \\
\text { Forest Classifier }\end{array}$ & 0.458 & 0.663 & 0.0486 & 0.044 \\
\hline $\begin{array}{l}\text { Super Learner ensemble and Decision } \\
\text { Tree Classifier }\end{array}$ & 0.629 & 0.600 & 0.025 & 0.025 \\
\hline $\begin{array}{l}\text { Super Learner ensemble and KNN } \\
\text { Classifier }\end{array}$ & 0.781 & 1.000 & 0.001 & 0.034 \\
\hline $\begin{array}{l}\text { Super Learner ensemble and Support } \\
\text { Vector Machine Classifier }\end{array}$ & 0.536 & 0.642 & 0.001 & 0.083 \\
\hline $\begin{array}{l}\text { Super Learner ensemble and Artificial } \\
\text { Neural Network Classifier }\end{array}$ & 0.111 & 0.249 & 0.093 & 0.007 \\
$\begin{array}{l}\text { Super Learner ensemble and Naïve } \\
\text { Bayes Classifier }\end{array}$ & 0.035 & 0.051 & 0.004 & 0.027 \\
\hline
\end{tabular}

When considering the $\mathrm{p}$ - values given by each algorithm pair in the prediction using LMS dataset, except the Naïve Bayes algorithm, all the other pairs were statistically proven that $\mathrm{H}_{0}$ couldn't be rejected since their $\mathrm{p}$ - values are greater than the minimum significant level. However, the $\mathrm{p}$ - value of Naïve Bayes vs Super Learner ensemble was 0.035 which is below the minimum significant level $(0.035<0.05)$, Therefore, the null hypothesis is rejected and H1 accepted, by statistically confirming that even before applying LLWSL approach both algorithms had performed differently. 
As illustrated in the above Table 6, the $\mathrm{p}$-values derived by each pair for LMS data after LLWSL, are below the significance level 0.05, which implies that all null hypothesis developed to test them are rejected by proving that there exists a significant difference between the individual performance of the base learner and the performance of the super learner ensemble.

The $\mathrm{p}$ - value analysis of each algorithm pair in the prediction using classroom dataset, has shown a similar pattern to LMS dataset. Except the p- value of Naïve Bayes vs Super Learner classifier, others have shown that $\mathrm{H}_{0}$ could not be rejected and the individual classifiers do not show a significantly different performance than the super learner ensemble before applying LLWSL. After applying LLWSL, the optimized ensemble has shown an improved prediction behavior except the p- value of support vector machine vs super learner, others are below the significance level 0.05 . Their null hypothesis is rejected by proving that the super learner behaves differently during the prediction task. According to the research findings, it is evident that, the super learner ensemble shows optimal performance.

\section{Discussion}

The researchers intend to further the discussion into another grooming technology called Deep Learning and compare the performance of the high performing super learner ensemble developed in this study with Convolutional Neural Network (CNN) technology [5]. Two separate CNNs were developed in TensorFlow and trained to classify the LMS dataset and the classroom dataset which were used to construct the super learner ensemble. According to the test results obtained, the CNN could obtain $42.5 \%$ of an accuracy in training the LMS dataset whereas the super learner could achieve $85 \%$ of an accuracy level. When the classroom dataset was trained by the CNN algorithm, it could obtain $63 \%$ of an accuracy while the super learner could achieve $69 \%$ of an accuracy. Although these accuracy measures are impressive on the part of the the super learner ensemble, external factors may 
affect the performance of deep learning CNN classifier. In most of the situations deep learning performs better in large volumes of data and even the goodness of the dataset has a large impact on the performance of deep learning [47]. A group of researchers have implemented a super learner to test it against a deep learning algorithm and they concluded that most of the times their deep learning classifier outperformed the super learner model [28].

In evaluating the super learner performance, two data sets were selected from the education domain. Students' interaction with a course module through online LMS system was available in LMSD dataset and the interactive classroom data was available in the classroom dataset. The number of instances in the data set are different, classroom dataset has 170 rows and the LMS Data file contains 799 records after preprocess the data. Both datasets consist of demographic, social and the performance details of the students. Researchers have mentioned that if the number of features/ dimensions in the dataset is less, the model is more accurate. If multiple dimensions are available in the training dataset, it will negatively impact the accuracies of some algorithms such as Support Vector Machine and Random Forest [25]. This leads to overfitting the classification algorithm; requires additional computational time and efforts such as preprocessing and develop relatively complex machine learning models [13]. Since the model derived by the algorithm depends on the behavior of the dataset, it is considerably difficult to produce evidence for the characteristics of the dataset which can interact negatively with the data and make low performance [24]. Generally, the machine learning algorithms are applied on the preprocessed, error free, noiseless and non- redundant data. Therefore, those obvious reasons do not affect the accuracies of algorithms of this study. The analysis commenced by performing feature selection as well. Therefore, the number of dimensions may not affect the performance of the machine learning algorithms.

As illustrated in the Table 4, it can be observed that no noteworthy variation exists between the accuracy between the super learner 
ensemble used for a dataset before and after LLWSL. Post LLWSL accuracies were also relatively closer to the Pre- LLWSL accuracies in each data set. This implies that the properties of the dataset have an impact on the accuracies of the algorithms. Once the feature selection is performed, the feature reduction has been done and resulted with similar number of features in both datasets. The number of instances can be considered as once such property which may have a direct impact on the performance. When comparing the LMS dataset with classroom data set, classroom dataset has fewer instances than LMS Data and the accuracy is lower than the model derived using LMS dataset.

The environment in which the experiment has been conducted can be considered as another property which may affect the performance of the accuracies of the super learner ensemble. However, the entire experiments were run on an Intel Core (R) i5 $72000 \mathrm{CPU} @ 2.7 \mathrm{GHz}$ machine with NVDIA TITAN GPU processor of which the prediction result was independent from the execution environment.

\section{Conclusion}

Rather than adhering to a model generated by a single machine learning algorithm, after a comparison of several machine learning models, an optimal super learner was implemented with improved accuracy and a high speed in generating the predicted model in this study. The model was tested using two main datasets in the education domain from several Sri Lankan universities. The prediction accuracy of the super learner model remained consistent though several changes were performed to the super learner model by the LLWSL algorithm. Moreover, a significantly greater execution time was shown by the super learner after the application of LLWSL algorithms. The proposed LLWSL algorithm was tested using two separate data sheets to validate the accuracy. After a successful validation process, it can be concluded that the proposed LLWSL algorithm results in an optimal super learner model. The study will be continued with more advanced 
hyperparameter optimization methods to obtain higher accuracy level and to apply the proposed approach to regression problems.

\section{Conflicts of Interest}

The authors declare no conflict of interest.

\section{Acknowledgement}

The authors would like to express their gratitude to University of Kelaniya and Sri Lanka Institute of Information Technology for the fullest support provided in gathering data for this study.

\section{References}

[1] O. I. Abiodun, A. Jantan, A. E. Omolara, K. V. Dada, N. A. Mohamed, H. Arshad. State-of-the-art in artificial neural network applications: A survey. Heliyon, 2018, 4, e00938.

[2] A. McCallum, K. Nigam. A comparison of event models for naïve bayes text classification, J. Mach. Learn. Res. 2003, 3, 1265-1287.

[3] K. Babalyan, R. Sultanov, E. Generozov, E. Sharova, E. Kostryukova, A. Larin, A., A. Kanygina, V. Govorun, G. Arapidi. LogLoss-BERAF: An ensemble-based machine learning model for constructing highly accurate diagnostic sets of methylation sites accounting for heterogeneity in prostate cancer. PloS One, 2018, 13, e0204371.

[4] M. B. Bardenet, K. Balázs, S. Michèle. Collaborative hyperparameter tuning. In Proceedings of the 30th International Conference on International Conference on Machine Learning. 2013,28, II-199-II-207.

[5] P. Bambharolia. Overview of Convolutional Neural Networks, 2017.

[6] A.P. Bradley. The use of the area under the ROC curve in the evaluation of machine learning algorithms. Pattern Recognit. 1997, 30, 1145-1159.

[7] L. Breiman, Bagging predictors. Mach. Learn. 1996, 24, 123-140

[8] G. Brown. Ensemble Learning. In C. Sammut and G. I. Webb (Eds.), Encyclopedia of Machine Learning and Data Mining Springer US, 2017, pp. 393-402

[9] D. Cheng, S. Zhang, Z. Deng, Y. Zhu, M. Zong. kNN Algorithm with Data-Driven k Value. 2014, 499-512.

[10] B. Clarke. Comparing Bayes model averaging and stacking when model approximation error cannot be ignored 2003, 4, 683-712.

[11] T. G. Dietterich. Ensemble Methods in Machine Learning. In: Multiple Classifier Systems. MCS 2000. Lecture Notes in Computer Science, Springer, 2000, 1857.

[12] R. Felder, R. Brent. Active learning: An introduction. ASQ Higher Education Brief. 2009, 2. 
[13] R. Genuer, J. M. Poggi, C. Tuleau-Malot. VSURF: An R Package for Variable Selection Using Random Forests. The R J. 2015, 7, 19-33.

[14] J. Bergstra, Y. Bengio. Random Search for Hyper-Parameter Optimization, J. Mach. Learn. Res. 2012, 13, 281- 305.

[15] T. Joachims. Text Categorization with Support Vector Machines: Learning with Many Relevant Features, In: European Conference on Machine Learning, Chemnitz, Germany, 1998, pp.137-142.

[16] E. Jones, T. Oliphant, P. Peterson. SciPy: Open source scientific tools for Python, 2001

[17] L. Julien-Charles,C. Gagné, R. Sabourin. Bayesian Hyperparameter Optimization for Ensemble Learning. 2016.

[18] J. W. Kim, B. H. Lee, M. J. Shaw, H. Chang and M. Nelson, Application of Decision Tree Induction Techniques to Personalized Advertisements on Internet Storefronts, Int. J. Electron. Commer. 2001, 5, 45-62.

[19] M. Kursa, W. Rudnicki. Feature selection with boruta package. J. Stat. Softw. 2010, $36,1-13$

[20] L. I. Kuncheva, C.J. Whitaker. Measures of Diversity in Classifier Ensembles and Their Relationship with the Ensemble Accuracy. Mach. Learn., 2003, 51, 181-207.

[21] M. Ladds, A. Thompson and J. Kadar, D. Slip, D. Hocking, R. Harcourt. Super machine learning: Improving accuracy and reducing variance of behaviour classification from accelerometry. Anim. Biotelemetry. 2017. 5, 1-9

[22] J. Large, J. Lines, A. Bagnall. A probabilistic classifier ensemble weighting scheme based on cross-validated accuracy estimate, Data Min. Knowl. Discov. 2019, 33, 1674-1709.

[23] C. Li, J. Wang, L. Hu, P. Gong. Comparison of classification algorithms and training sample sizes in urban land classification with landsat thematic mapper imagery. Remote Sens. 2014, 6, 964-983

[24] F. Löw, F., U. Michel, S. Dech, C. Conrad. Impact of feature selection on the accuracy and spatial uncertainty of per-field crop classification using support vector machines. ISPRS J. Photogramm. and Remote Sens., 2013, 85, 102-119.

[25] M. Pal, G. M. Foody. Feature selection for classification of hyperspectral data by svm. IEEE Transactions on Geoscience and Remote Sensing, 2010, 48, 2297-2307.

[26] F. Pedregosa, G. Varoquaux, A. Gramfort, V. Michel, B. Thirion, O. Grisel, et al. Scikit-learn: Machine learning in Python. J. Mach. Learn. Res. 2011, 12, 2825-2830

[27] P. K. Douglas, S. Harris, A. Yuille, M. S. Cohen. Performance comparison of machine learning algorithms and number of independent components used in fMRI decoding of belief vs. disbelief, NeuroImage, 2011, 56, 544-553

[28] S. Purushotham, C. Meng, Z. Che, Y. Liu. Benchmark of Deep Learning Models on Large Healthcare MIMIC Datasets. J. Biomed. Inform. 2017, 83, 112-134 
[29] R. Markovic, S. Wolf, J. Cao, E. Spinnräker, D. Wölki, J. Frisch, C. van Treeck. Comparison of different classification algorithms for the detection of user's interaction with windows in office buildings, Energy Procedia, 2017, 122, 337-342

[30] H.C. Romesburg. Cluster analysis for researchers. Melbourne, 2014, FL: Krieger.

[31] M. Re, G. Valentini. Ensemble methods: A review. 2012.

[32] K.T. S. Kasthuriarachchi, S. R. Liyanage, C. M. Bhatt. A data mining approach to identify the factors affecting the academic success of tertiary students in sri lanka. In: Caballé S., Conesa J. (eds) software data engineering for network elearning environments. lecture notes on data engineering and communications technologies, 2018, 11. Springer, Cham

[33] M. Shahhosseini, G. Hu, H. Pham. Optimizing ensemble weights for machine learning models: a case study for housing price prediction. 2020.

[34] H. Shee, K. Cheruiyot,S. Kimani. Application of k-nearest neighbor classification in medical data mining, 2014, 4 .

[35] R. Sherri. Mortality risk score prediction in an elderly population using machine learning, Am. Journal of Epidemiology, 2013, 177, 443-452.

[36] S. Soheily-Khah, Y. Wu. Ensemble learning using frequent itemset mining for anomaly detection. 2018.

[37] S. Sperandei. Understanding logistic regression analysis. Biochemia Medica. 2014.

[38] I. Syarif, E. Zaluska, A. Prugel-Bennett, G. Wills. Application of bagging, boosting and stacking to intrusion detection. 2012.

[39] T. G. Dietterich. An experimental comparison of three methods for constructing ensembles of decision trees: Bagging, boosting and randomization. Mach. Learn. 2000, 40, 139-158.

[40] J. Vamathevan, D. Clark, P. Czodrowski, I. Dunham, E. Ferran, G. Lee, B. Li, A. Madabhushi, P. Shah, M. Spitzer, S. Zhao. Applications of machine learning in drug discovery and development. Nature reviews. Drug Discov. 2019, 18, 463 477.

[41] H. R. Varian. Big Data: New Tricks for Econometrics. J. Econ. Perspect. 2014, 28, 328.

[42] S. Wang, J. Tang, H. Liu. Feature Selection. 2016.

[43] J. Wong, T. Manderson, M. Abrahamowicz, D. Buckeridge, R. Tamblyn. Can hyperparameter tuning improve the performance of a super learner: a case study. Epidemiology. 2019.

[44] D. H. Wolpert. Stacked generalization. Neural Networks, 1992, 5,241-259

[45] D. Wolpert, W. Macready. No free lunch theorems for search 1996.

[46] D. Yogatama, G. Mann. Efficient transfer learning method for automatic hyperparameter tuning. AISTATS., 2014.

[47] G. Zhou, K. Sohn, H. Lee. Online incremental feature learning with denoising 
autoencoders. In: International Conference on Artificial Intelligence and Statistics. JMLR.org. 2014, 1453-1461

[48] J. Demsar. Statistical comparisons of classifiers over multiple data sets, J. Mach. Learn. Research, 2007 ,7, 1-30.

[49] C. Nadeau, Y. Bengio. Inference for the generalization error, J. Mach. Learn. 2003, $52,239-281$ 